\title{
Correction to: Spatial chromatin architecture alteration by structural variations in human genomes at the population scale
}

Michal Sadowski ${ }^{1,2}$, Agnieszka Kraft ${ }^{1,3}$, Przemyslaw Szalaj ${ }^{1,4,5}$, Michal Wlasnowolski ${ }^{1,3}$, Zhonghui Tang ${ }^{6}$, Yijun Ruan ${ }^{7^{*}}$ and Dariusz Plewczynski ${ }^{1,3^{*}}$ (i)

Correction to: Sadowski et al. Genome Biol (2019) 20:148

https://doi.org/10.1186/s13059-019-1728-x

Following publication of the original article [1], it was noticed that the incorrect Fig. 2 and Fig. 3. were processed during production. It was also noticed that Fig. 4a was processed with a superfluous " $1 \mathrm{e} 7$ " symbol in the upper right corner.

The corrected Fig. 2, Fig. 3 and Fig. 4 are provided below. The original article has been corrected. The publishers apologize for the error.

\footnotetext{
Author details

${ }^{1}$ Centre of New Technologies, University of Warsaw, Banacha 2c, 02-097

Warsaw, Poland. '2Faculty of Physics, University of Warsaw, Pasteura 5, 02-093

Warsaw, Poland. ${ }^{3}$ Faculty of Mathematics and Information Science, Warsaw University of Technology, Koszykowa 75, 00-662 Warsaw, Poland. ${ }^{4}$ Centre for Innovative Research, Medical University of Bialystok, Kilinskiego 1, 15-089

Bialystok, Poland. ${ }^{5}$-BioStat, Hasselt University, Agoralaan building D, BE3590, Diepenbeek, Belgium. 'Zhongshan School of Medicine, Sun Yat-sen

University, Guangzhou 510080, China. ${ }^{7} T$ The Jackson Laboratory for Genomic Medicine, 10 Discovery Drive, Farmington, CT 06032, USA.
}

Published online: 03 September 2019

\section{Reference}

1. Sadowski, et al. Spatial chromatin architecture alteration by structural variations in human genomes at the population scale. Genome Biol. 2019; 20:148. https://doi.org/10.1186/s13059-019-1728-x.

\footnotetext{
* Correspondence: Yijun.Ruan@jax.org; d.plewczynski@cent.uw.edu.pl

${ }^{7}$ The Jackson Laboratory for Genomic Medicine, 10 Discovery Drive,

Farmington, CT 06032, USA

${ }^{1}$ Centre of New Technologies, University of Warsaw, Banacha 2c, 02-097

Warsaw, Poland

Full list of author information is available at the end of the article
}

(c) The Author(s). 2019 Open Access This article is distributed under the terms of the Creative Commons Attribution 4.0 International License (http://creativecommons.org/licenses/by/4.0/), which permits unrestricted use, distribution, and reproduction in any medium, provided you give appropriate credit to the original author(s) and the source, provide a link to the Creative Commons license, and indicate if changes were made. The Creative Commons Public Domain Dedication waiver (http://creativecommons.org/publicdomain/zero/1.0/) applies to the data made available in this article, unless otherwise stated. 
A

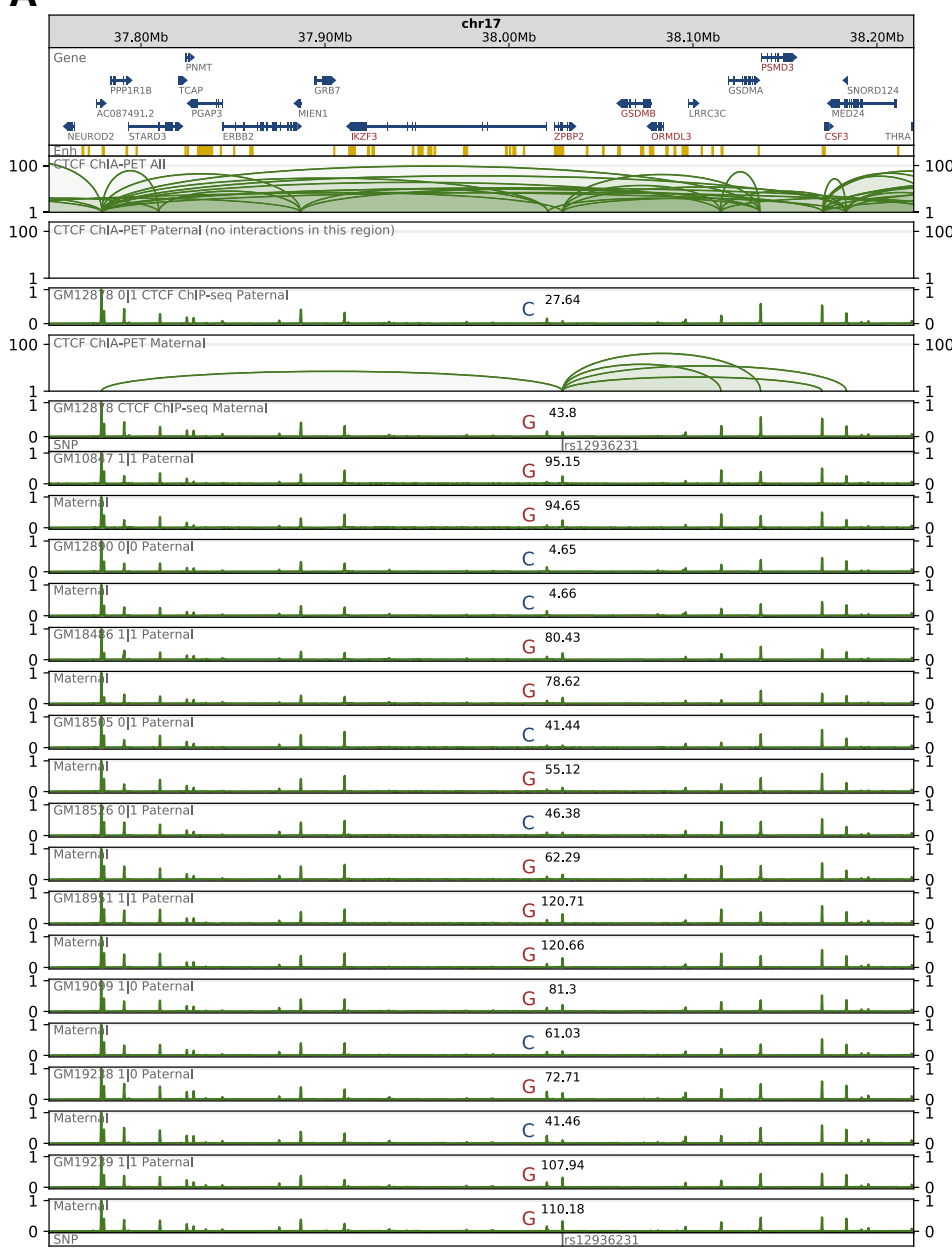

B

100 .

C
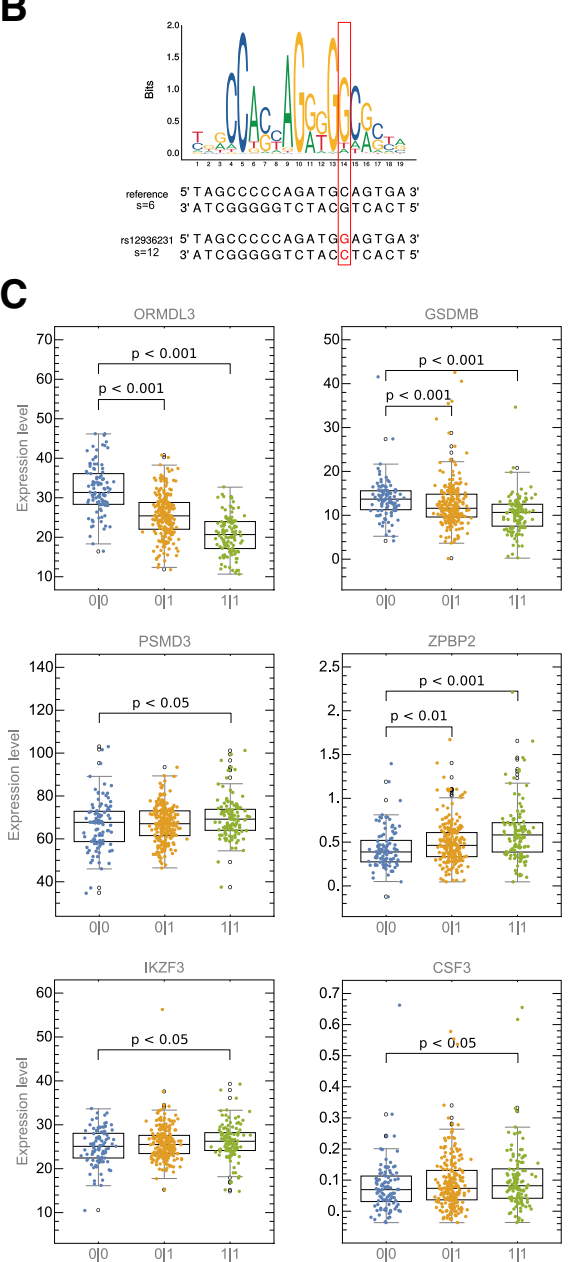

$\mathbf{E}$
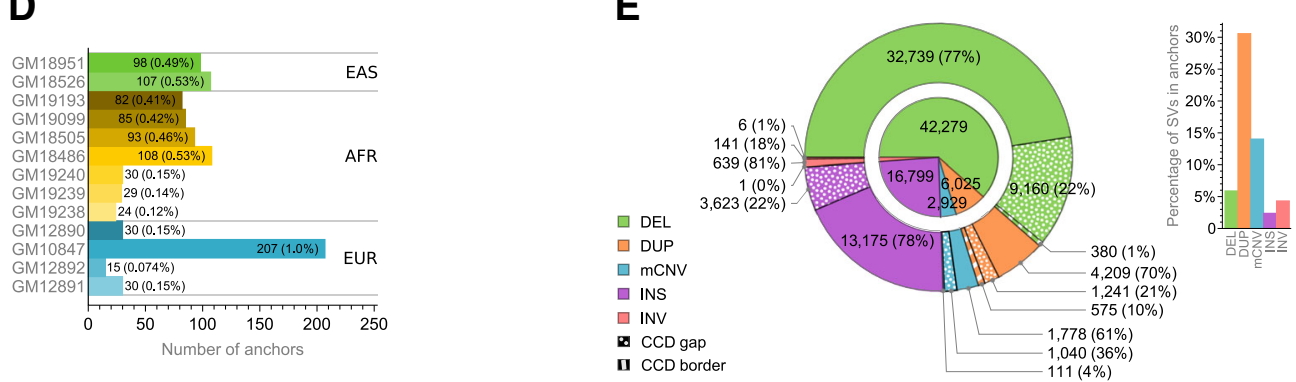

Fig. 2 Predicting the impact of SVs on the chromatin topology. a Browser view of a 0.5-Mb genomic segment with asthma-associated SNP rs12936231 identified in a part of the human population. SNP rs12936231 alters the sequence of a CTCF motif involved in interactions. Haplotypespecific CTCF signals from 10 lymphoblastoid cells are presented along with haplotype-specific CTCF ChIA-PET interactions from GM12878 (only a subset of all interactions can be identified as specifically paternal/maternal as it is done based on allele-specific SNPs emerging at the interaction anchors). For each track, ChIP-seq signal values (originally in RPMs) were divided by the maximal value of the signal in the visualized region. Sum of the signal values over the genomic region occupied by the SNP-affected interaction anchor together with the genotype is marked in each signal track. $\mathbf{b}$ Comparison of sequences and scores of CTCF binding motifs carrying the reference C and the alternative G alleles of rs 12936231 . c Differences in gene transcription rates between genotypes set for rs12936231. Genes exhibiting differences in transcription which pass MannWhitney test with $p$ value $<0.05$ were reported. Center lines show the medians; box limits indicate the 25 th and 75 th percentiles; whiskers extend 1.5 times the interquartile range (IQR) from the 25th and 75th percentiles; outliers are represented by rings; far outliers (points beyond 3 times the IQR) are not represented by any element of box plots. $n=101,227,117$ sample points. $\mathbf{d}$ CTCF anchors from GM12878 not intersected with CTCF ChIP-seq peaks identified in different lymphoblastoid cells. The anchors were filtered by consensus CTCF binding sites (see the "Methods" section). e Number of SVs, divided by type, intersecting (in case of interaction anchors), covering (in case of CCD boundaries), or contained in (in case of CCDs and CCD gaps) different genomic structural elements 


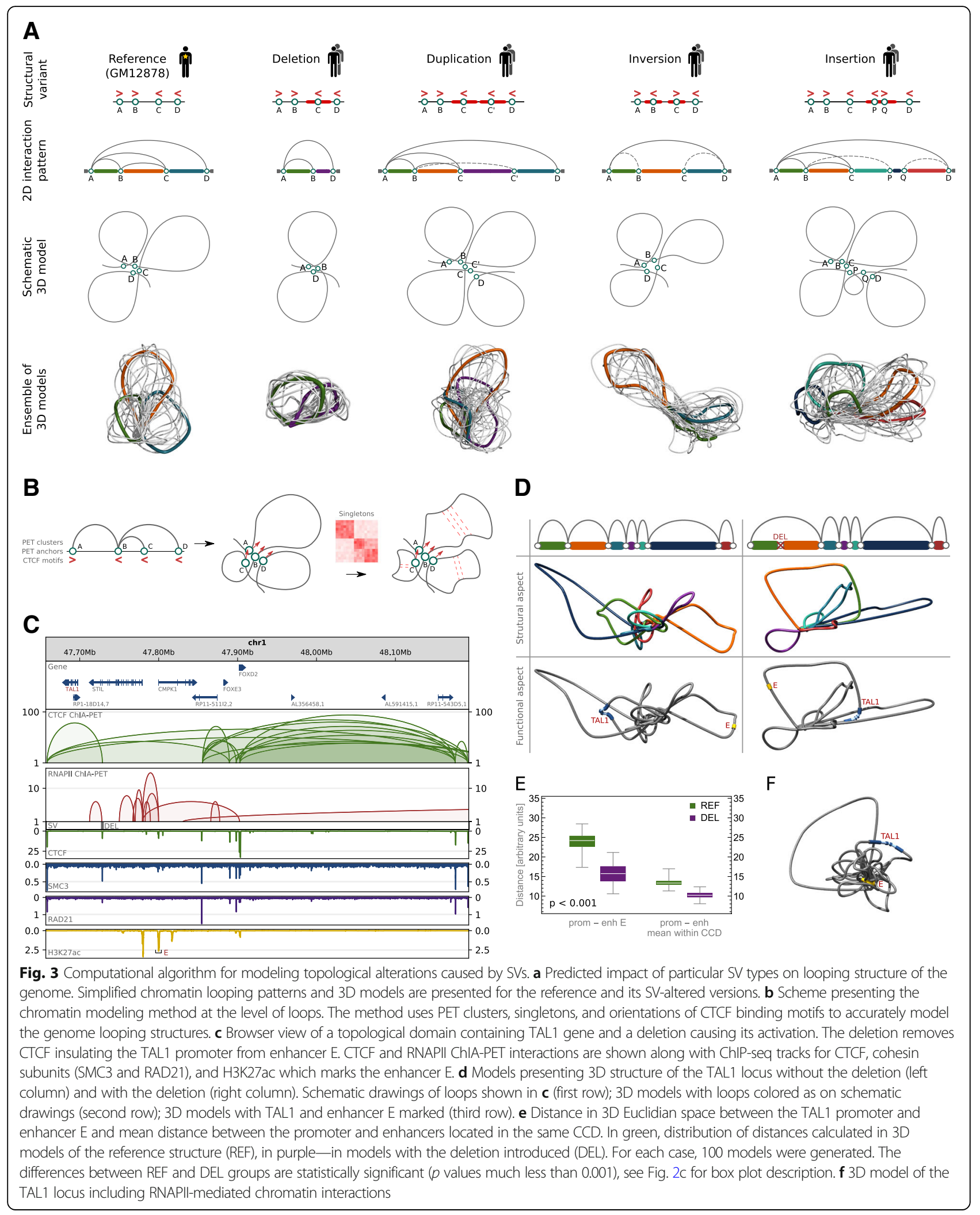



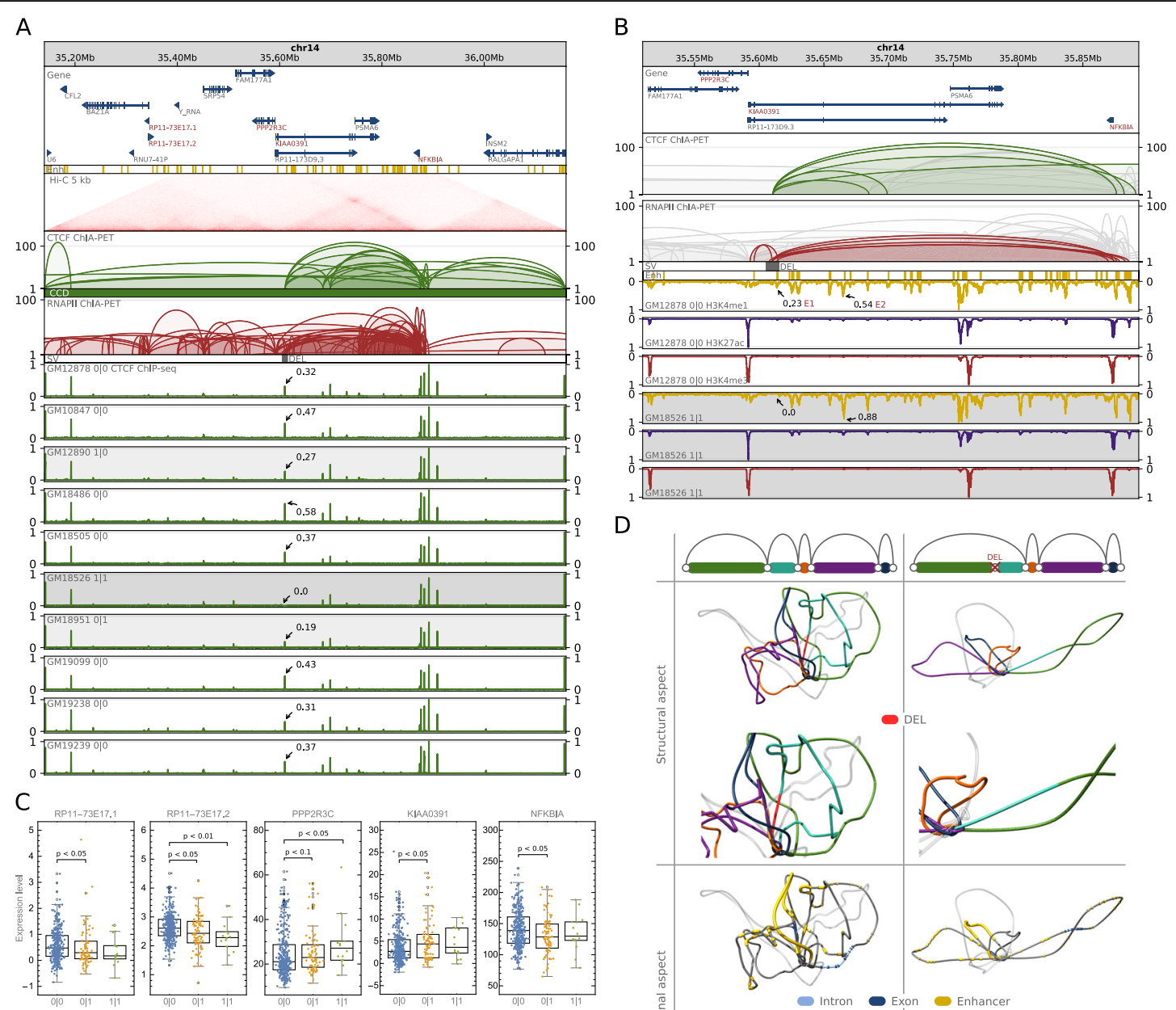

D

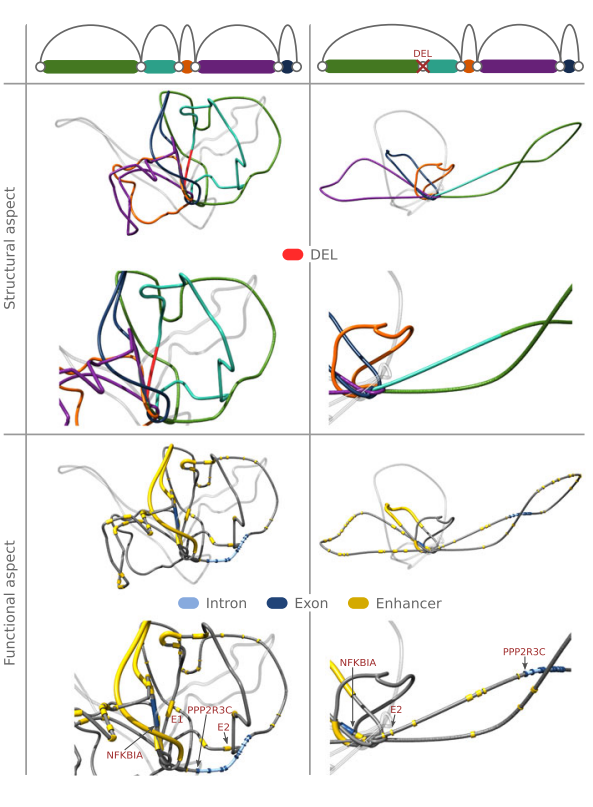

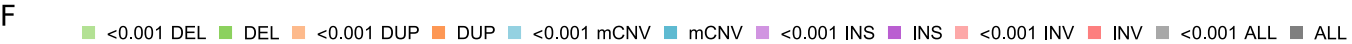
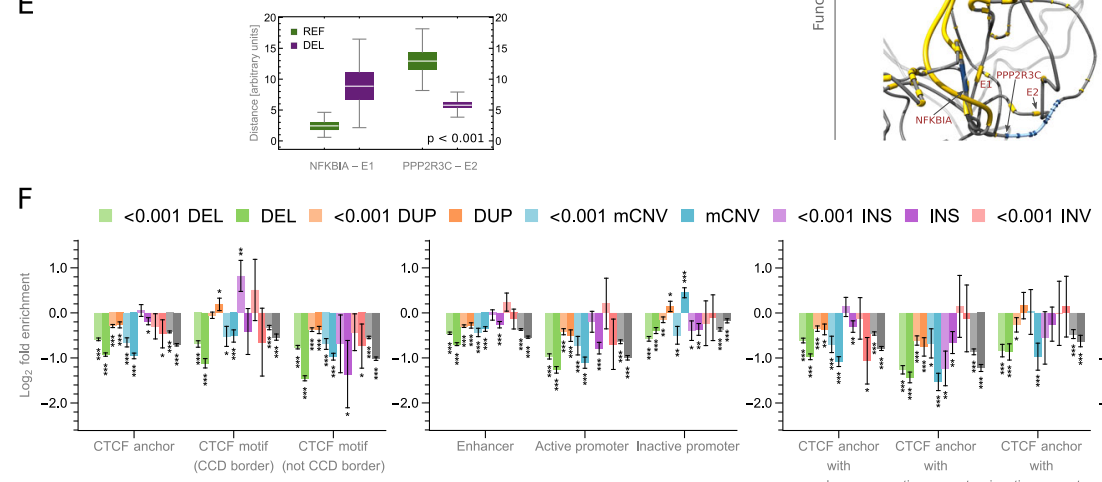

CTCF anchor CTCF anchor CTCF anchor
with
with

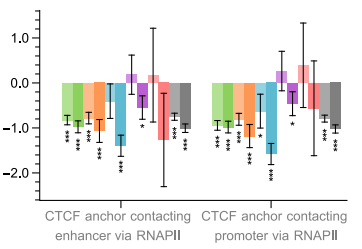

G
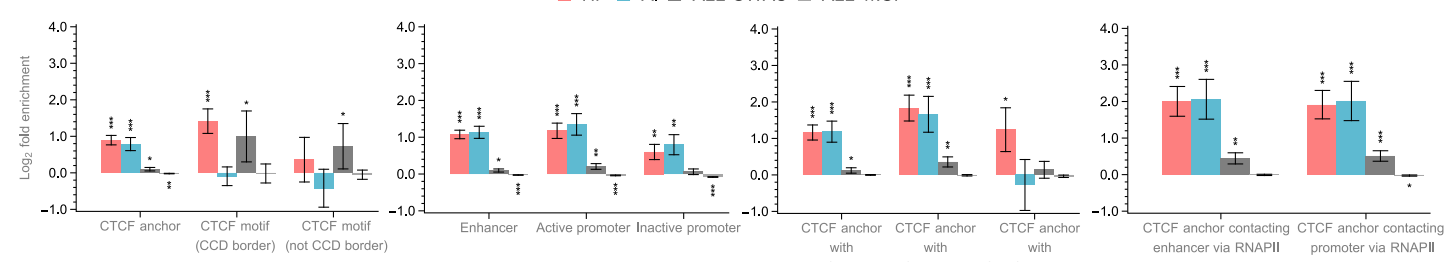

Fig. 4 (See legend on next page.) 
(See figure on previous page.)

Fig. 4 Impact of SVs on genome organization at the population scale. a Browser view of a 1-Mb genomic segment with a deletion identified in a part of the human population. The deletion removes a CTCF anchor with enhancer located in an intron of KIAA0391. CTCF ChIP-seq signals from 10 lymphoblastoid cells of different genotypes are presented for comparison. For each track, ChIP-seq signal values (originally in RPMs) were divided by the maximal value of the signal in the visualized region. The highest signal peak in the genomic region covered by the deletion is marked in each signal track. $\mathbf{b}$ Close-up on ChIA-PET interactions at the deletion site displayed above the ChIP-seq profiles of histone modifications for GM12878 - no deletion and GM18526 - homozygous deletion. H3K4me1 is primarily associated with active enhancers, H3K27ac - with active promoters and enhancers, H3K4me3-with promoters. Compare with Additional file 2: Fig. S7. c Differences in gene transcription rates between genotypes defined by the deletion. Genes exhibiting the differences in transcription which pass Mann-Whitney test with $p$ value $<0.1$ were reported, see Fig. 2 for box plot description. $n=346,85,14$ sample points. $\mathbf{d}$ 3D models of the domain shown in a without the deletion (left column) and with the deletion (right column). Schematic drawings of loops shown in $\mathbf{b}$ (first row); 3D models with loops colored as on schematic drawings (second row); 3D models with NFKBIA and PPP2R3C genes (arrows are pointing toward the TSSs) and enhancers marked (third row). Every picture has its duplicated zooming in on the deletion site. e Distance in 3D Euclidean space between the NFKBIA promoter and enhancer E1 and between the PPP2R3C promoter and enhancer E2. In green, distribution of distances calculated in 3D models of the reference structure (REF), in purple-in models with the deletion introduced (DEL). For each case, 100 models were generated. The differences between REF and DEL groups are statistically significant ( $p$ values much less than 0.001 ), see Fig. $2 c$ for box plot description. $\mathbf{f}$ Enrichment/depletion of genomic structural elements with SVs of different types and of different VAF (VAF $<0.001$ and VAF $\geq 0.001)$. In case of CCD borders, only these fully imbedded in SV intervals are counted as affected, whereas for other structural elements $\geq 1$ bp overlaps are counted. Error bars represent SD. $\mathbf{g}$ Enrichment/depletion of genomic structural elements with the 1000 Genomes Project SNPS (ALL 1kGP), all GWAS SNPS (ALL GWAS), GWAS SNPs associated with hematological parameters (HP), and with autoimmune diseases (Al). Error bars represent SD 